\title{
A description and estimate of very low-intensity activity and inactive awake time in community-dwelling adults with chronic spinal cord injury
}

\author{
M-J Perrier and KA Martin Ginis, The SHAPE-SCI Research Group
}

Study Design: Secondary analysis of cross-sectional data.

Objectives: To estimate the amount of very low-intensity activity (VLPA) or inactive daily awake time that people with spinal cord injury $(\mathrm{SCl})$ engage in and to determine correlates of VLPA/inactivity in this population.

Setting: Community (Ontario, Canada).

Methods: Participants with $\mathrm{SCl}\left(n=695 ; M_{\mathrm{age}}=47\right.$ years; $M_{\text {years post-injury }}=15$ years, $76 \%$ men) completed telephone interviews. Demographic details and injury-related characteristics were self-reported. Total daily physical activity (for example, mild, moderate and heavy intensity) was measured using the Physical Activity Recall Assessment for People with SCI (PARA-SCI). VLPA/inactivity was calculated by subtracting total daily physical activity time from daily awake time. Correlates of VLPA/inactivity were assessed using a hierarchical linear regression where demographic variables were entered on the first step and injury-related characteristics were entered second.

Results: Participants reported VLPA/inactivity for approximately $768 \pm 169$ min per day, or $84 \%$, of their awake time. The regression model predicting VLPA/inactivity was not significant.

Conclusion: People with $\mathrm{SCl}$ spend the majority of their awake time in VLPA/inactivity. However, VLPA/inactivity did not differ as a function of demographic or injury-related variables, suggesting that all segments of the SCl population could benefit from strategies to reduce inactivity.

Spinal Cord (2016) 54, 709-713; doi:10.1038/sc.2015.232; published online 19 January 2016

\section{INTRODUCTION}

People with spinal cord injuries (SCI) have a greater risk of several negative health outcomes when compared with people in the general population. Post-injury changes to body composition, such as increased fat mass, and metabolic changes are associated with an elevated risk of chronic disease such as metabolic syndrome, Type II diabetes and cardiovascular disease. ${ }^{1,2}$ Furthermore, people with SCI have a greater risk of mortality from chronic disease when compared with people in the general population. ${ }^{3}$ For example, people with SCI have 3.6 times higher risk of dying from cardiovascular disease and 2.6 times greater risk of death from diabetes when compared with people in the general population. ${ }^{3}$ As such, knowledge of the behavioural risk factors for chronic disease and ultimately how to reduce risk among this population is needed.

Physical activity is a behaviour that can mitigate the risk of developing many chronic diseases. In a review of exercise and sport among people with SCI, support was shown for the relationship between greater leisure-time physical activity (LTPA) and lower cardiovascular risk factors including lower BMI, fat mass, waist circumference, insulin resistance and C-reactive protein. ${ }^{4}$ Furthermore, the review also highlighted evidence from training studies that found a positive effect of LTPA, specifically body-weight-supported treadmill training, on glucose tolerance and insulin sensitivity. ${ }^{4}$
Given the profound impairments in physical functioning that typically follow an SCI, most men and women either reduce or completely stop participating in LTPA post injury. Not surprisingly, people with SCI accrue substantially less daily LTPA than the general population; this observation has been noted across different contexts and settings. ${ }^{5,6}$ For example, Martin Ginis and colleagues ${ }^{7}$ reported that only $50 \%$ of people with SCI accrue any LTPA. Tasiemski et al. ${ }^{8}$ have found similar results, with an estimated $47 \%$ of their sample engaging in some LTPA. This stands in stark contrast to estimates in the general population where approximately $75-88 \%$ of people report engaging in at least some LTPA. ${ }^{9}$

In addition to LTPA, a few studies have explored the relationship between other forms of health and fitness and physical activity, such as activities of daily living (ADL) similar to wheeling for transportation and household chores. For example, Hetz et al. ${ }^{10}$ found that more time spent wheeling as an ADL was associated with better health and fitness. Despite the possible benefits of engaging in physically active ADL, Rimmer et al. ${ }^{11}$ noted that people with disabilities are less likely to engage in these types of unstructured physical activities throughout the day when compared with people without disabilities. They also noted that people with specific disability characteristics, such as wheelchair users, have limited opportunities for both spontaneous and structured physical activities and may be more likely to spend 
their time sitting and engaged in very low-intensity activity (VLPA)/inactivity. ${ }^{11}$ As such, further exploration of VLPA/inactivity, its correlates and health outcomes is warranted.

In the general population, a growing body of literature has shown associations between increased bouts of inactive sitting time and increases in cardiometabolic and inflammatory markers of chronic disease including insulin resistance and C-reactive protein. ${ }^{12}$ Moreover, research has shown that, even among adults without disabilities who are meeting the physical activity guidelines, greater sitting time is associated with these chronic disease markers. ${ }^{13}$ As such, it has been suggested that inactivity and prolonged sitting time may represent unique risk factors for chronic disease risk, independent of the role played by LTPA. ${ }^{13}$ Despite these associations in the general population, little is known about VLPA/inactivity among people with SCI.

Given that only about half of the SCI population participates in any LTPA, ${ }^{7,8}$ and even fewer are presumed to engage in other forms of physical activity, ${ }^{11}$ it is likely that people with SCI spend considerable

Table 1 VLPA/inactivity (in minutes) as a function of demographic and injury-related characteristics

\begin{tabular}{|c|c|c|c|}
\hline Variable & N (\%) & Mean (s.d.) & $\begin{array}{c}\% \text { Time in } \\
\text { VLPA/inactivity }\end{array}$ \\
\hline \multicolumn{4}{|l|}{ Sex } \\
\hline Female & $164(23.60 \%)$ & $753.10(157.16)$ & 83.27 \\
\hline Male & 531 (76.40\%) & 773.48 (171.17) & 83.91 \\
\hline \multicolumn{4}{|l|}{ Education } \\
\hline High school & 241 (34.98\%) & 773.05 (152.98) & 84.92 \\
\hline College & 169 (24.53\%) & 764.14 (177.90) & 82.99 \\
\hline University & $121(17.56 \%)$ & 759.98 (180.78) & 81.65 \\
\hline Postgraduate & $45(6.53 \%)$ & $767.75(164.45)$ & 83.51 \\
\hline Other & 113 (16.40\%) & 777.95 (176.54) & 84.83 \\
\hline \multicolumn{4}{|l|}{ Ethnicity } \\
\hline Caucasian & $616(89.28 \%)$ & 768.03 (168.33) & 83.64 \\
\hline Native Canadian & $17(2.46 \%)$ & 727.30 (161.94) & 85.65 \\
\hline Black & $21(3.04 \%)$ & 844.76 (168.96) & 87.62 \\
\hline Asian & $16(2.32 \%)$ & 782.88 (161.92) & 83.89 \\
\hline Other & $20(2.90 \%)$ & 744.79 (175.86) & 82.29 \\
\hline \multicolumn{4}{|l|}{ Marital status } \\
\hline Single & 246 (35.76\%) & $782.28(167.82)$ & 84.97 \\
\hline Married & 305 (44.33\%) & 760.06 (166.14) & 82.93 \\
\hline Common law & $36(5.23 \%)$ & 761.02 (178.25) & 83.15 \\
\hline Divorced & 86 (12.50\%) & 775.95 (180.92) & 83.76 \\
\hline Widowed & $15(2.18 \%)$ & 737.72 (126.56) & 84.20 \\
\hline \multicolumn{4}{|l|}{ Injury severity } \\
\hline C1-C4, ASIA A-C & 75 (10.93\%) & 766.63 (166.32) & 84.61 \\
\hline C5-C8, ASIA A-C & $184(26.82 \%)$ & 748.93 (178.07) & 82.43 \\
\hline T1-S5, ASIA A-C & 255 (37.17\%) & 775.83 (161.60) & 84.96 \\
\hline ASIA D, Any Level & 172 (25.07\%) & 777.35 (170.03) & 82.56 \\
\hline \multicolumn{4}{|l|}{ Mode of mobility } \\
\hline Manual chair & 389 (55.97\%) & 775.22 (168.69) & 83.73 \\
\hline Power chair & 221 (31.80\%) & 746.32 (171.94) & 83.35 \\
\hline Gait aid & 85 (12.23\%) & 796.82 (149.11) & 84.94 \\
\hline
\end{tabular}

Abbreviation: ASIA, American Spinal Injury Association; VLPA, very low-intensity activity. Note: Not all participants answered the demographic questions, $n<695$ for some demographics. time inactive or engaged in VLPA. Therefore, the first objective of this study was to estimate the proportion of time that people with SCI spend engaging in VLPA/inactivity during their waking hours. The second objective was to determine correlates of VLPA/inactivity. Given that past studies have identified age, sex, years post-injury and injury severity to be correlates of LTPA among people with $\mathrm{SCI}^{7,14}$ and age and sex have been correlated with sedentary behaviour in the general population, ${ }^{15}$ we hypothesised that age, sex, years post-injury and injury severity would be significantly associated with VLPA/inactivity. Marital status, ethnicity and education are not associated with LTPA among people with $\mathrm{SCI}^{7,14}$ and inconsistently associated with sedentary behaviour in the general population. ${ }^{15}$ As such, we hypothesised that these demographics would not be associated with VLPA/inactivity.

\section{MATERIALS AND METHODS}

This study is a secondary analysis of data from the Study of Health and Activity in People with Spinal Cord Injury (SHAPE-SCI). A full description of the SHAPE-SCI study design and participants has been published. ${ }^{16}$ As a brief summary, SHAPE-SCI is a prospective cohort study of 695 communitydwelling adults with traumatic SCI. Participants were recruited from four centres across the province of Ontario and were required to (1) reside in Ontario, (2) be over the age of 18, (3) have a traumatic SCI, (4) require mobility assistance outside of their homes and (5) understand and speak English. Participants were recruited in several ways, including lists of former SCI patients interested in participating in research, advertisements in SCI-relevant publications and by word of mouth. ${ }^{16}$ Prior to the telephone interview, participants were required to provide voluntary, informed consent. The Research Ethics Boards at all four centres approved materials and methods.

For this analysis, all 695 participants who completed a baseline interview were included. Participants were representative of the Canadian SCI population, ${ }^{17,18}$ with an average age of 47 years (s.d. $=13$ years) and 15 years post injury (s.d. $=11$ years). Seventy-six percent were male and $51 \%$ were paraplegic. Demographics are presented in Table 1.

\section{Measures}

VLPA and inactivity. Participants' physical activity was measured using the Physical Activity Recall Assessment for People with SCI (PARA-SCI). The PARA-SCI was created specifically for assessing physical activity among people with SCI. The interview schedule was developed in consultation with both rehabilitation specialists and people with SCI and structured by eight specific periods (for example, morning routine, breakfast, morning and so on) and detailed flowcharts and questions were used to aid participant recall. ${ }^{19,20}$ All interviewers received formal trainer and were required to complete a mock interview prior to data collection to ensure quality. During the interviews, participants reported all physical activities they performed over the previous 3 days including ADL (for example, chores) and LTPA (for example, sport). The intensity of each activity was rated as mild, moderate, heavy or nothing at all. As per the PARA-SCI administration protocol, only activities rated as mild or greater are recorded. Research using the PARA-SCI suggests that these are activities with a metabolic equivalent (MET) value of 1.5 or greater. ${ }^{21}$ Participants were also asked to report the times they woke up and went to sleep each day. Average daily awake time was created by taking a sum of daily awake time and dividing by three. Daily physical activity was calculated by creating a sum of the minutes spent in mild, moderate and heavy intensity physical activity (for example, both LTPA and ADL) and dividing by three. VLPA/inactivity was calculated by subtracting minutes of daily physical activity from minutes of daily awake time. Percentage of VLPA/inactivity was calculated by dividing the minutes of VLPA/inactivity by minutes of total awake time and multiplying by 100 . The PARA-SCI has demonstrated reliability and validity for use in people with SCI, establishing validity through comparison with both doubly labelled water and indirect calorimetry. ${ }^{19,21,22}$

Demographic characteristics. Participants reported their age, sex, education, ethnicity and marital status at baseline. All categorical variables were assessed with fixed response options and dummy coded prior to analysis. 
Injury-related characteristics. At baseline, participants reported years postinjury, injury severity and mode of mobility. Years post injury were calculated as the number of years between the injury date and the baseline interview. Injury severity was a composite variable composed of the level of injury and the American Spinal Injury Association Impairment Scale (AIS) score. Injury severity was then coded into four groups according to the International SCI Core Data Set's recommendations. ${ }^{23}$ Specifically, participants with high tetraplegia and an AIS score of A, B or C formed the first group. Participants with low tetraplegia and an AIS score of A, B or C formed the second group. Participants with paraplegia and an AIS score of A, B or C formed the third group. Participants with an AIS score of D formed the final group. Participants were asked to identify the primary mode of mobility they used outside of the home, which was classified as a manual chair, power chair or a gait aid (for example, walker, cane).

\section{Data management and analysis}

Data were scanned for missing values, outliers and normality prior to analysis. Eleven participants were missing values for daily awake time; given that this value was $<5 \%(1.5 \%)$, these values were imputed using mean replacement. ${ }^{24}$ Outliers were defined as values greater than the absolute $Z$-score of 3.29 and were replaced using the highest value that was not an outlier. Twelve, 16 and 12 outliers were replaced for mild, moderate and heavy intensity physical activity, respectively. Seven outliers were replaced for daily awake time.

Descriptive statistics, including mean, median and binning into quartiles, were calculated for participants' VLPA/inactivity. To determine whether any demographic or injury-related variables were associated with the percentage of VLPA/inactivity, hierarchical linear regression was used. Demographic variables were entered in the first block, and injury-related variables were entered in the second block. Statistical significance was determined by an alpha set at 0.05 . All analyses were completed in SPSS Statistics v.20 IBM Corp, Armonk, NY, USA.

\section{RESULTS}

Participants accrued approximately $148 \mathrm{~min}$ per day (s.d.=135) of total physical activity, with $121 \mathrm{~min}$ per day (s.d. $=127)$ spent on ADL and $27 \mathrm{~min}$ per day spent on LTPA, as previously reported by Martin Ginis et al. ${ }^{7}$ Participants spent $768 \mathrm{~min}$ per day $($ s.d. =169) on VLPA/inactivity and were awake for $916 \mathrm{~min}$ per day (s.d.=103). As such, participants were engaged in VLPA/inactivity for $84 \%$ of their waking minutes (25th Percentile $\mathrm{VLPA}_{\mathrm{V} \text { inactivity }}=622 \mathrm{~min}$ per day, $76 \%$; 50th Percentile VLPA/inactivity $=789 \mathrm{~min}$ per day, $88 \%$; 75th Percentile $\mathrm{VLPA}_{\mathrm{V} \text { inactivity }}=889 \mathrm{~min}$ per day, 95\%). Figure 1 demonstrates the percentage of time spent on LTPA, ADL and VLPA/inactivity.

VLPA/inactivity as a function of demographic and injury-related characteristics is displayed in Table 1 . The regression model (Table 2)



Figure 1 Breakdown of daily awake time: ADL, LTPA and VLPA/inactivity. Mean minutes of $A D L=27.14$ (2.98\%), $L T P A=121.33 \quad(13.41 \%)$, VLPA $=767.85(83.65 \%)$. was not significant in Block 1, $F(14661)=1.03, P=0.42, R^{2}=0.02$; the addition of injury-related characteristics in Block 2 did not significantly increase the variance explained, $F(20,655)=0.99$, $P=0.47, \Delta R^{2}=0.00$.

\section{DISCUSSION}

The purpose of this study was to estimate the amount of VLPA/inactivity, and possible correlates, among people with SCI. Participants engaged in VLPA/inactivity for an average of $13 \mathrm{~h}$ per day, or $\sim 84 \%$, of their awake time. Contrary to our hypothesis and the findings from studies of predictors of LTPA, the regression model was not significant; none of the demographic or injury-related predictors (for example, age, sex, injury severity, years post-injury and mode of mobility) were significant. ${ }^{7}$ One possible explanation for these results may reflect the use of a combined physical activity variable that accounts for all LTPA and ADL. It is possible that the differences between subgroups on VLPA/inactivity were masked by the differences between groups with respect to time spent on ADLs and LTPA. Consider sex as an example. Research on overall LTPA, as well as specific forms of LTPA such as sport, routinely shows that men accrue more minutes of LTPA than women. ${ }^{7}$ However, emerging literature

Table 2 Hierarchical regression model for VLPA/inactivity

\begin{tabular}{|c|c|c|c|c|c|}
\hline $\begin{array}{l}\text { Predictor } \\
(\text { constant }=80.48)\end{array}$ & $B$ & $S E$ & $\beta$ & $\begin{array}{l}95 \% \text { Cl for B } \\
\text { (lower, upper) }\end{array}$ & $\Delta \mathrm{R}^{2}$ \\
\hline Block 1 & & & & & - \\
\hline Age & $0.13^{*}$ & 0.05 & 0.11 & $0.03,0.23$ & \\
\hline Sex & 0.55 & 1.41 & 0.02 & $-2.21,3.32$ & \\
\hline \multicolumn{6}{|l|}{ Education } \\
\hline College & -1.58 & 1.57 & -0.05 & $-4.66,1.50$ & \\
\hline University & -2.88 & 1.74 & -0.07 & $-6.29,0.55$ & \\
\hline Postgraduate & -1.61 & 2.49 & -0.03 & $-6.51,3.28$ & \\
\hline Other & -0.78 & 1.79 & -0.02 & $-4.29,2.73$ & \\
\hline \multicolumn{6}{|l|}{ Ethnicity } \\
\hline Native Canadian & -0.26 & 3.91 & -0.00 & $-7.93,7.41$ & \\
\hline Black & 3.09 & 3.41 & 0.04 & $-3.60,9.79$ & \\
\hline Asian & 1.08 & 4.00 & 0.01 & $-6.77,8.92$ & \\
\hline Other & -0.55 & 3.46 & -0.01 & $-7.35,6.25$ & \\
\hline \multicolumn{6}{|l|}{ Marital status } \\
\hline Married & $-3.43^{*}$ & 1.47 & -0.11 & $-6.30,-0.55$ & \\
\hline Common law & -1.89 & 2.80 & -0.03 & $-7.38,3.61$ & \\
\hline Divorced & -2.93 & 2.01 & -0.06 & $-6.88,1.02$ & \\
\hline Widowed & -2.81 & 4.30 & -0.03 & $-11.25,5.63$ & \\
\hline Block 2 & & & & & 0.00 \\
\hline \multicolumn{6}{|l|}{ Injury severity } \\
\hline $\mathrm{C} 1-\mathrm{C} 4, \mathrm{ASIA} \mathrm{A}-\mathrm{C}$ & 3.89 & 2.57 & 0.08 & $-1.16,8.94$ & \\
\hline C5-C8, ASIA A-C & 1.43 & 1.94 & 0.04 & $-2.37,5.24$ & \\
\hline T1-S5, ASIA A-C & 3.22 & 1.83 & 0.10 & $-0.37,6.82$ & \\
\hline \multicolumn{6}{|l|}{ Mode of mobility } \\
\hline Power chair & -1.25 & 1.52 & -0.04 & $-4.23,1.73$ & \\
\hline Gait aid & 2.06 & 2.34 & 0.04 & $-2.54,6.66$ & \\
\hline Years post-injury & -0.00 & 0.06 & -0.00 & $-0.12,0.11$ & \\
\hline
\end{tabular}

Abbreviation: VLPA, very low-intensity activity.

Note: VLPA/inactivity conceptualised as a percentage of daily awake time. Referent categories for the categorical variables are high school (education), Caucasian (ethnicity), single (marital status), ASIA D (injury severity) and manual chair (mode of mobility). 
concerning ADLs suggests that women spend more time on specific activities, such as preparing meals and cleaning, when compared with men. ${ }^{10}$ Thus, the lack of association between sex and VLPA/inactivity may be due to the different types of physical activities pursued.

Past research supports an association between injury severity, mode of mobility and LTPA. Yet contrary to hypotheses, no association was found between injury severity and VLPA/inactivity or mode of mobility and VLPA/inactivity. Given that both time spent on ADLs and LTPA was accounted for, it is possible that participation in ADLs can make up for any differences between groups with respect to LTPA participation. For example, certain ADLs such as wheeling or cleaning may not be as intense or time-consuming for participants with greater function. With respect to ADLs, Hetz et al. ${ }^{10}$ found that individuals with tetraplegia reported spending twice as much time toileting and dressing when compared with people with paraplegia. As such, it is possible that participants with greater function report a similar amount of time spent in VLPA/inactivity when compared with participants with less function because they are more efficient with their ADLs and have more time to spend in LTPA, as well as VLPA/inactivity. Furthermore, Kozlowski and Heinneman ${ }^{25}$ found that, across all injury severity classes, factors such as the number of physiotherapy treatments were related to function at discharge and 2 years later. Moreover, a recent study found that wheelchair skills, as opposed to function, are also related to social integration and community participation. ${ }^{26}$ Thus, participants with greater confidence in their skill, regardless of injury severity, may be less likely to spend time in VLPA/inactivity given opportunities to spend time in recreation and other activities. In consideration of these findings and the past literature, it may not be as simple as exploring the relationship between demographics, injury-related characteristics and VLPA/inactivity, but rather exploring environmental (for example, access to rehabilitation) and psychosocial (for example, self-efficacy) determinants that will reduce VLPA/inactivity. Further investigation of the determinants of VLPA/inactivity is warranted.

This study is the first to provide a population-based estimate of VLPA/inactivity in individuals with SCI. Nevertheless, a few limitations warrant mention. First, although the PARA-SCI has been validated against objective measures of physical activity, ${ }^{18}$ there is a risk of self-report bias in people's reports of physical activity. Future studies exploring VLPA/inactivity should consider using more objective measures of energy expenditure when feasible. Second, although our sample is representative of Canadians with SCI, there remains a risk of volunteer bias as those who contacted the study team with interest may be different than those who did not. Third, not all of the categories in each demographic variable were equal, leading to wide $95 \%$ confidence intervals for some estimates. Further research that remedies these imbalances may be necessary. Finally, as a means of understanding the extent of the disparity in VLPA/inactivity between people with SCI and the general population, research that uses objective estimates of VLPA/inactivity will enable direct comparisons with current estimates for the general population.

Although our study has highlighted the extent of VLPA/inactivity behaviours among people with SCI, VLPA/inactivity was measured as the absence of mild, moderate and heavy physical activity. Further research is needed to examine the metabolic and health implications of performing different types of VLPA/inactivity, such as watching TV versus occupational activities. Further research is also needed to examine the temporal patterns of VLPA/inactivity. As Owen et al. ${ }^{13}$ demonstrated in the general population, the negative effects of inactivity may be mitigated by breaking up inactive time.

\section{CONCLUSION}

People with SCI spend the majority of their awake time in VLPA/inactivity. Previously identified correlates of LTPA, such as injury severity, are not associated with VLPA/inactivity. As such, traditional predictors of physical activity may not provide clinicians with the information needed to tailor and target future interventions to reduce VLPA/inactivity. Given that VLPA/inactivity may be an independent contributor to health, further research is necessary to better understand and form the foundation for interventions to reduce VLPA/inactivity among people with SCI.

\section{DATA ARCHIVING}

There were no data to deposit.

\section{CONFLICT OF INTEREST}

The authors declare no conflict of interest.

1 Cragg JJ, Noonan VK, Dvorak M, Krassioukov A, Mancini GBJ, Borisoff JF. Spinal cord injury and type 2 diabetes: results from a population health survey. Neurology 2013; 81: 1864-1868.

2 Myers J, Lee M, Kiratli J. Cardiovascular disease in spinal cord injury: an overview of prevalence, risk, evaluation, and management. Am J Phys Med Rehabil 2007; 86: $1-11$.

3 Garshick E, Kelley A, Cohen SA, Garrison A, Tun CG, Gagnon D et al. A prospective assessment of mortality in chronic spinal cord injury. Spinal Cord 2005; 43: 408-416.

4 Martin Ginis KA, Jorgensen S, Stapleton J. Exercise and sport for persons with spinal cord injury. Phys Med Rehabil 2012; 4: 894-900.

5 van den Berg-Emons RJ, Bussmann JB, Haisma JA, Sluis TA, van der Woude LH, Bergen MP et al. A prospective study on physical activity levels after spinal cord injury during inpatient rehabilitation and the year after discharge. Arch Phys Med Rehabil 2008; 89: 2094-2101.

6 Buchholz AC, McGillivray CF, Pencharz PB. Physical activity levels are low in free-living adults with chronic paraplegia. Obes Res 2003; 11: 563-570.

7 Martin Ginis KA, Latimer AE, Arbour-Nicitopoulos KP, Buchholz AC, Bray SR, Craven $\mathrm{BC}$ et al. Leisure time physical activity in a population-based sample of people with spinal cord injury, part 1: Demographic and injury-related correlates. Arch Phys Med Rehabil 2010; 91: 722-728.

8 Tasiemski T, Bergstrom E, Savic G, Gardner BP. Sports, recreation and employment following spinal cord injury: a pilot study. Spinal Cord 2000; 38: 173-184.

9 Colley RC, Garriguet D, Janssen I, Craig CL, Clarke J, Tremblay MS. Physical activity of Canadian adults: accelerometer results from the 2007 to 2009 Canadian Health Measures Survey. Health Rep 2011; 22: 7-14.

10 Hetz S, Latimer AE, Martin Ginis KA. Activities of daily living performed by individuals with SCl: Relationships with physical fitness and leisure time physical activity. Spinal Cord 2009; 47: 550-554.

11 Rimmer JH, Schiller W, Chen MD. Effects of disability-associated low energy expenditure deconditioning syndrome. Exerc Sport Sci Rev 2012; 40: 22-9.

12 Healy GN, Matthews CE, Dunstan DW, Winkler EA, Owen N. Sedentary time and cardio-metabolic biomarkers in US adults: NHANES 2003-06. Eur Heart J 2011; 32: 590-597.

13 Owen N, Healy GN, Matthews CE, Dunstan DW. Too much sitting: The populationhealth science of sedentary behavior. Exerc Sport Sci Rev 2010; 38: 105-113.

14 Perrier MJ, Latimer-Cheung AE, Martin Ginis KA, SHAPE-SCI Research Group. An investigation of seasonal variation in leisure-time physical activity in persons with spinal cord injury. Spinal Cord 2012; 50: 507-511.

15 Rhodes RE, Mark RS, Temmel CP. Adult sedentary behavior: a systematic review. Am J Prev Med 2012; 42: e3-e28.

16 Martin Ginis KA, Latimer AE, Buchholz AC, Bray SR, Craven BC, Hayes KC et al. Establishing evidence-based physical activity guidelines: Methods for the Study of Health and Activity in People with Spinal Cord Injury (SHAPE SCI). Spinal Cord 2008; 46: $216-221$

17 Noonan VK, Fingas M, Farry A, Baxter D, Singh A, Fehlings MG et al. Incidence and prevalence of spinal cord injury in Canada: A national perspective. Neuroepidemiology 2012; 39: 219-226.

18 Farry A, Baxter D. The Incidence and Prevalence of Spinal Cord Injury in Canada: Overview and Estimates Based on Current Evidence. Rick Hansen Institute Urban Futures Institute: Vancouver, Canada, 2010.

19 Martin Ginis KA, Latimer AE, Hicks AL, Craven BC. Development and evaluation of an activity measure for people with spinal cord injury. Med Sci Sports Exerc 2005; 37: 1099-1111.

20 Martin Ginis KA, Latimer AE. Physical Activity Recall Assessment for People with Spinal Cord Injury: Administration and scoring manual. McMaster University: Hamilton, ON, Canada, 2008. 
21 Tanhoffer RA, Tanhoffer AIP, Raymond J, Hills AP, Davis GM. Comparison of methods to assess energy expenditure and physical activity in people with spinal cord injury. J Spinal Cord Med 2012; 35: 35-45.

22 Latimer AE, Martin Ginis KA, Craven BC, Hicks AL. The Physical Activity Recall Assessment for People with Spinal Cord Injury: Validity. Med Sci Sports Exerc 2006; 38: 208-216.

23 DeVivo M, Biering-Sørensen F, Charlifue S, Noonan V, Post M, Stripling T et al. International Spinal Cord Injury Core Data Set. Spinal Cord 2006; 44: 535-540.
24 Tabachnick BG, Fidell LS. Using multivariate statistics. Pearson/Allyn \& Bacon: Boston, MA, USA, 2007.

25 Kozlowski AJ, Heinemann AW. Using individual growth curve models to predict recovery and activities of daily living after spinal cord injury: An SCIRehab project study. Arch Phys Med Rehabil 2013; 94: S154-S164.

26 Smith EM, Sakakibara BM, Miller WC. A review of factors influencing participation in social and community activities for wheelchair users. Disabl Rehabil Assist Technol 2014; 4: 1-14. 